\title{
Dirac Magnons in Honeycomb Ferromagnets
}

\author{
Sergey S. Pershoguba, ${ }^{1}$ Saikat Banerjee, ${ }^{1,2,3}$ J. C. Lashley, ${ }^{2}$ Jihwey Park, ${ }^{4}$ Hans Ågren, ${ }^{3}$ \\ Gabriel Aeppli, ${ }^{4,5,6}$ and Alexander V. Balatsky ${ }^{1,2,7}$ \\ ${ }^{1}$ Nordita, Center for Quantum Materials, KTH Royal Institute of Technology, and Stockholm University, \\ Roslagstullsbacken 23, S-106 91 Stockholm, Sweden \\ ${ }^{2}$ Institute for Materials Science, Los Alamos National Laboratory, Los Alamos, New Mexico 87545, USA \\ ${ }^{3}$ Division of Theoretical Chemistry and Biology, Royal Institute of Technology, \\ SE-10691 Stockholm, Sweden \\ ${ }^{4}$ Paul Scherrer Institute, CH-5232 Villigen PSI, Switzerland \\ ${ }^{5}$ Laboratory for Solid State Physics, ETH Zurich, Zurich CH-8093, Switzerland \\ ${ }^{6}$ Institut de Physique, EPF Lausanne, Lausanne CH-1015, Switzerland \\ ${ }^{7}$ Department of Physics, University of Connecticut, Storrs, Connecticut 06269, USA
}

(Received 11 June 2017; published 23 January 2018)

\begin{abstract}
The discovery of the Dirac electron dispersion in graphene [A. H. Castro Neto, et al., The Electronic Properties of Graphene, Rev. Mod. Phys. 81, 109 (2009)] led to the question of the Dirac cone stability with respect to interactions. Coulomb interactions between electrons were shown to induce a logarithmic renormalization of the Dirac dispersion. With a rapid expansion of the list of compounds and quasiparticle bands with linear band touching [T. O. Wehling, et al., Dirac Materials, Adv. Phys. 63, 1 (2014)], the concept of bosonic Dirac materials has emerged. We consider a specific case of ferromagnets consisting of van der Waals-bonded stacks of honeycomb layers, e.g., chromium trihalides $\mathrm{CrX}_{3}(\mathrm{X}=\mathrm{F}, \mathrm{Cl}, \mathrm{Br}$ and I), that display two spin wave modes with energy dispersion similar to that for the electrons in graphene. At the single-particle level, these materials resemble their fermionic counterparts. However, how different particle statistics and interactions affect the stability of Dirac cones has yet to be determined. To address the role of interacting Dirac magnons, we expand the theory of ferromagnets beyond the standard Dyson theory [F. J. Dyson, General Theory of Spin-Wave Interactions, Phys. Rev. 102, 1217 (1956), F. J. Dyson, Thermodynamic Behavior of an Ideal Ferromagnet, Phys. Rev. 102, 1230 (1956)] to the case of nonBravais honeycomb layers. We demonstrate that magnon-magnon interactions lead to a significant momentum-dependent renormalization of the bare band structure in addition to strongly momentumdependent magnon lifetimes. We show that our theory qualitatively accounts for hitherto unexplained anomalies in nearly half-century-old magnetic neutron-scattering data for $\mathrm{CrBr}_{3}$ [W. B. Yelon and $\mathrm{R}$. Silberglitt, Renormalization of Large-Wave-Vector Magnons in Ferromagnetic $\mathrm{CrBr}_{3}$ Studied by Inelastic Neutron Scattering: Spin-Wave Correlation Effects, Phys. Rev. B 4, 2280 (1971), E. J. Samuelsen, et al., Spin Waves in Ferromagnetic $\mathrm{CrBr}_{3}$ Studied by Inelastic Neutron Scattering, Phys. Rev. B 3, 157 (1971)]. We also show that honeycomb ferromagnets display dispersive surface and edge states, unlike their electronic analogs.
\end{abstract}

DOI: 10.1103/PhysRevX.8.011010

\section{INTRODUCTION}

The observation of fermionic quasiparticles with Dirac dispersion was a key finding for graphene [1]. Since then, the list of materials exhibiting the Dirac and Weyl energy spectra for fermions has been extended further. Materials hosting

Published by the American Physical Society under the terms of the Creative Commons Attribution 4.0 International license. Further distribution of this work must maintain attribution to the author(s) and the published article's title, journal citation, and DOI.
Subject Areas: Graphene, Magnetism, Topological Insulators

bosons characterized by Dirac cones have opened a new stage in the investigation of Dirac materials such as photonic crystals [2,3], plasmonic systems [4], honeycomb arrays of superconducting grains [5], and magnets [6,7]. Magnets studied in the distant past [8,9], especially in the form of transition metal trihalides $\mathrm{TMX}_{3}$ (where $\mathrm{X}=\mathrm{F}, \mathrm{Cl}, \mathrm{Br}, \mathrm{I}$, and $\mathrm{TM}=\mathrm{Cr}$ ), consist of weakly coupled honeycomb ferromagnetic planes. These materials have a potential as spin-polarizing elements and exhibit strong Kerr and Faraday effects.

Early spin-wave analysis revealed a Dirac crossing point in the dispersion in the honeycomb layers containing two 
magnetic atoms per unit cell. Neutron-scattering measurements revealed hitherto unexplained anomalies $[8,9]$ in the boson (spin-wave) self-energies near the Dirac points.

The single-particle properties for both bosonic and fermionic Dirac materials derive from the tight-binding model on the honeycomb lattice and are, thus, identical. At the level of quantum statistics, however, there is a difference between Dirac fermions and bosons. For fermions, the excitations occur near the chemical potential, and one can focus on the low-energy Dirac cones shown in Fig. 1(a). In contrast, bosons are not subject to the Pauli exclusion principle. They can explore the entire momentum space, and excitations near zero energy dominate at low temperatures.

The importance of many-body effects was appreciated immediately for fermionic Dirac materials [10]. In particular, the Coulomb interaction between electrons [see Fig. 1(c)] leads to a logarithmic renormalization of the Dirac cone velocity. This renormalization was verified by observing an anomalous dependence of the cyclotron frequency on the carrier concentration [11].

A similar analysis for interacting bosons and their effects on the Dirac node dispersion has not been done systematically, to our knowledge. Since the early work by Bloch [12], the physics of magnons has remained a subject of active experimental and theoretical research [13-15]. Despite an immense number of works, the important case

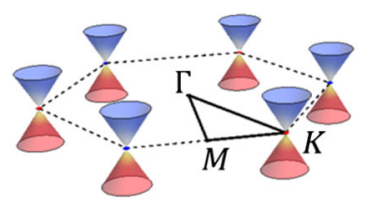

(a)

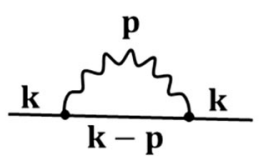

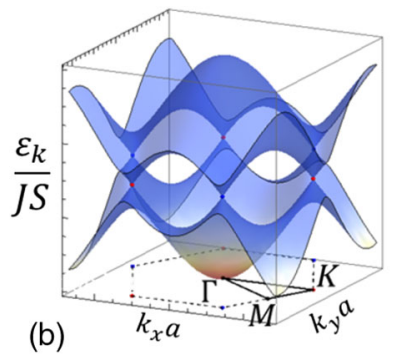

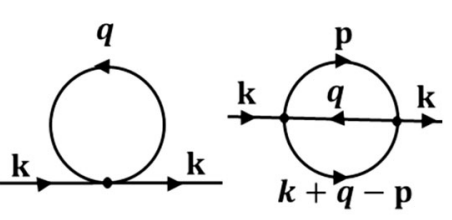

(d) $\quad \Sigma \sim T^{n}$
FIG. 1. Comparison of Dirac fermions (left panels) vs bosons (right panels). (a) The properties of the Dirac fermion materials are determined by the states at the chemical potential. So, approximating the band structure by the Dirac cones near the zone centers is sufficient. (b) In contrast, since bosons can freely occupy any states within the Brillouin zone, information about the entire band structure is necessary. In the top panels, the color scheme (red to blue) is an artistic representation of the particle occupation number from strong to weak. Temperature can efficiently excite the bosons in the down-band in the vicinity of the $\Gamma$ point. (c) The relevant self-energy diagram that gives the logarithmic correction to the Dirac fermion velocity. (d) The relevant self-energy diagrams giving a temperature-dependent renormalization and decay of the Dirac bosons. of ferromagnets with non-Bravais lattices has received relatively little attention. In the two milestone papers by Dyson [16,17], magnon thermodynamics is discussed only for crystals with Bravais lattices. Therefore, such theories cannot be directly applied to the honeycomb lattice, a prominent example of non-Bravais bipartite lattices.

Here we focus on Dirac bosons, as realized for example by $\mathrm{Cr}$ trihalides with ferromagnetic honeycomb lattices. We start with a two-dimensional (2D) honeycomb ferromagnet, where the bosons are spin waves (magnons) that form Dirac nodes, Fig. 1(b). We calculate the lowest-order self-energy diagrams shown in Fig. 1(d). The Hartree self-energy gives a uniform renormalization of the energy bands consistent with the theory of Bloch [18]. We then consider both the real and imaginary parts of the self-energy, which give the energy renormalization and decay rate of magnon excitations. We find that interactions induce strong temperature and momentum-dependent renormalization of the magnon bands near a Dirac node. Our results resolve the outstanding puzzle of spin-wave anomalies seen in $\mathrm{CrBr}_{3}$ [8,9].

We also discuss the band structure and the role of topology in a three-dimensional (3D) model of $\mathrm{CrBr}_{3}$. We note that topological magnonic systems have received great attention recently [6,7,19-23]. A number of these systems are gapped by the Dzyaloshinskii-Moriya interaction $[7,19,20]$, producing nontrivial Haldane topology and chiral edge states, which are protected against backscattering and could, thus, be used for spintronic applications. In contrast, we are interested in the topology of gapless systems. We show that $\mathrm{CrBr}_{3}$ is the highly soughtafter magnetic analog of $\mathrm{ABC}$ stacked graphite. In this system, the Dirac nodal line $[21,24-26]$ is protected by a combination of orbital time-reversal and inversion symmetries. The nodal line has an associated topological $\pi$ Berry phase winding, which induces surface states [24-26]. We identify the difference between the fermionic and magnonic surface states in $\mathrm{ABC}$-stacked honeycomb lattices.

Our results are applicable to the rapidly growing class of van der Waals ferromagnets with non-Bravais lattices. We note that recent experimental works by Gong et al. [27] and Huang et al. [28] demonstrate the robustness of the honeycomb ferromagnetic phase in few-layer van der Waals materials including $\mathrm{CrI}_{3}$. Recent theoretical works $[29,30]$ with a focus on antiferromagnets are also relevant for our discussion.

The outline of the paper is as follows. After this introduction, we present the free spin-wave theory in Sec. II. In Sec. III, we present the main result of the paper-the evaluation of the self-energies and their effect on the magnon spectrum. In Sec. IV, we discuss properties of topological surface states, and we conclude in Sec. V.

\section{MODEL}

We begin with a Heisenberg model on a 2D honeycomb lattice with two sites per unit cell commonly denoted as $A$ and $B$. The Heisenberg Hamiltonian of the model 
$\mathcal{H}=-J \sum_{\langle i j\rangle} \boldsymbol{S}_{i} \cdot \boldsymbol{S}_{j}$ describes the nearest-neighbor coupling between spins. For convenience, we choose energy units for the coupling $J$, whereas the magnitude of spin $S$ is dimensionless. Positive coupling $J>0$, together with small Ising anisotropy and/or interplanar couplings, implies a ferromagnetic ground state at low temperatures $\left(T_{c}=32.5 \mathrm{~K}, S=\right.$ $3 / 2$ for $\mathrm{CrBr}_{3}$ ). We choose to study the magnetic excitations, the Dirac magnons, above the preexisting ferromagnetic ground state on the honeycomb lattice. For the moment, we ignore the easy-axis anisotropy of the chromium trihalides [31], as well as the interlayer coupling in $\mathrm{CrBr}_{3}$, which will be discussed at the end of the paper.

We follow standard practice and bosonize the Heisenberg Hamiltonian using the Holstein-Primakoff transformation. Truncated to zeroth order, these transformations relate the spin operators to the magnon creation or annihilation operators as $S^{x}+i S^{y}=\sqrt{2 S} a, S^{x}-i S^{y}=$ $\sqrt{2 S} a^{\dagger}$, and $S^{z}=S-a^{\dagger} a$. We thus obtain the free bosonic Hamiltonian

$$
\mathcal{H}=\sum_{\mathbf{k}} \Psi_{\mathbf{k}}^{\dagger} H_{0}(\mathbf{k}) \Psi_{\mathbf{k}}, \quad H_{0}=J S\left(\begin{array}{cc}
3 & -\gamma_{k} \\
-\gamma_{k}^{*} & 3
\end{array}\right) .
$$

Here, the two-by-two single-particle Hamiltonian $H_{0}$ acts upon the spinor $\Psi_{\mathbf{k}}=\left(a_{\mathbf{k}} b_{\mathbf{k}}\right)^{T}$ with components corresponding to the two sublattices. The off-diagonal element $\gamma_{k}=\sum_{j} e^{i k r_{j}}$ is ubiquitous for the honeycomb lattice and corresponds to coupling along the three nearest-neighbor inplane bond vectors $\mathbf{r}_{j}$. The energy spectrum of the single particle consists of two branches: the acoustic "down" and optical "up" branches with the dispersion $\varepsilon_{\mathbf{k}}^{u, d}=J S\left(3 \pm\left|\gamma_{\mathbf{k}}\right|\right)$ and wave functions $\Psi_{\mathbf{k}}^{u, d}=\left(e^{i \phi_{\mathbf{k}} / 2} s e^{-i \phi_{\mathbf{k}} / 2}\right) / \sqrt{2}$, where $s=-1(s=+1)$ for the $u(d)$ state and the momentumdependent phase $\phi_{\mathbf{k}}=\arg \gamma_{\mathbf{k}}$ is introduced. The magnon energy dispersion is plotted in Fig. 1(b). The down branch touches zero energy quadratically in the center of the Brillouin zone near the $\Gamma$ point as $\varepsilon_{\mathbf{k}}^{d}=3 J S k^{2} / 4$. The gapless magnons at the $\Gamma$ point are protected by the Goldstone theorem. The magnon branches have symmetry-protected Dirac crossings at the $K$ and $K^{\prime}$ points of the Brillouin zone characteristic of the honeycomb lattice. At the single-particle level, the magnon dispersion law is identical to the energy spectrum of electrons in graphene.

\section{RESULTS: BULK STATES}

The interaction vertex is obtained from the next-order terms following from the Holstein-Primakoff transformation (see Ref. [32] for details)

$$
\begin{aligned}
\mathcal{V}= & \frac{J}{4 N} \sum_{\left\{k_{i}\right\}} \gamma_{\boldsymbol{k}_{2}}^{*} a_{\boldsymbol{k}_{1}}^{\dagger} b_{\boldsymbol{k}_{2}}^{\dagger} a_{\boldsymbol{k}_{3}} a_{\boldsymbol{k}_{4}}+\gamma_{\boldsymbol{k}_{4}} a_{\boldsymbol{k}_{1}}^{\dagger} a_{\boldsymbol{k}_{2}}^{\dagger} a_{\boldsymbol{k}_{3}} b_{\boldsymbol{k}_{4}} \\
& +\gamma_{\boldsymbol{k}_{2}} b_{\boldsymbol{k}_{1}}^{\dagger} a_{\boldsymbol{k}_{2}}^{\dagger} b_{\boldsymbol{k}_{3}} b_{\boldsymbol{k}_{4}}+\gamma_{\boldsymbol{k}_{4}}^{*} b_{\boldsymbol{k}_{1}}^{\dagger} b_{\boldsymbol{k}_{2}}^{\dagger} b_{\boldsymbol{k}_{3}} a_{\boldsymbol{k}_{4}} \\
& -4 \gamma_{\boldsymbol{k}_{4}-\boldsymbol{k}_{2}} a_{\boldsymbol{k}_{1}}^{\dagger} b_{\boldsymbol{k}_{2}}^{\dagger} a_{\boldsymbol{k}_{3}} b_{\boldsymbol{k}_{4}},
\end{aligned}
$$

where the momentum before and after scattering is conserved, $\mathbf{k}_{1}+\mathbf{k}_{2}=\mathbf{k}_{3}+\mathbf{k}_{4}$. Note that the same function $\gamma_{\mathbf{k}}$ defined above for the single-particle Hamiltonian Eq. (1) also occurs in the interaction term Eq. (2).

We analyze the effect of interactions to first order and evaluate the Hartree diagram in Fig. 1(d). For simplicity, consider the case of low temperature, $T \ll J$, where only the low-energy down-magnons with momenta close to $\mathbf{q}=\mathbf{0}$ are excited. The Hartree term corresponds to contracting a pair of boson operators in Eq. (2) and replacing them with the thermodynamic boson occupation number of the magnons in the lower band, $\left\langle d_{\mathbf{q}}^{\dagger} d_{\mathbf{q}}\right\rangle=$ $f\left(\varepsilon_{\mathbf{q}}^{d}\right)=\left[\exp \left(\varepsilon_{\boldsymbol{q}}^{d} / T\right)-1\right]^{-1}$. We further perform a lowtemperature expansion and rewrite the self-energy,

$$
\Sigma_{k}^{(1)}(T)=-\alpha_{1} T^{2} H_{0}(\mathbf{k}),
$$

where $\alpha_{1}=\left[\pi /\left(24 \sqrt{3} J^{2} S^{3}\right)\right]$. The matrix elements of the two-by-two Hamiltonian are renormalized by the same temperature-dependent ratio $\alpha_{1} T^{2}$. This is a consequence of a delicate balance between the bare spectrum and the interaction term, both containing the honeycomb function $\gamma_{\mathbf{q}}$. So, the energy of the renormalized bands becomes $\left(1-\alpha_{1} T^{2}\right) \varepsilon_{\mathbf{k}}^{u, d}$. The magnon-magnon interaction leads to an overall temperature-dependent bandwidth renormalization. This is consistent with the observation by Bloch [18], who discussed a similar effect for a single-band case on a cubic lattice.

Next, we analyze the effect of interactions to second order. We consider the sunset diagram in Fig. 1(d),

$$
\Sigma_{\mathbf{k}}^{(2)}(T)=A^{2} \int \frac{d^{2} q d^{2} p}{(2 \pi)^{4}} \frac{\left|V_{\mathbf{k}, \mathbf{q} ; \mathbf{p}}\right|^{2} f\left(\varepsilon_{\boldsymbol{q}}\right)}{\varepsilon_{\boldsymbol{k}}+\varepsilon_{\mathbf{q}}-\varepsilon_{\mathbf{p}}-\varepsilon_{\mathbf{k}+\mathbf{q}-\mathbf{p}}+i \delta},
$$

where $V_{\mathbf{k}, \mathbf{q} ; \mathbf{p}}$ is the scattering matrix element, and $A=$ $3 \sqrt{3} a^{2} / 2$ is the area of the honeycomb lattice unit cell. Let us comment on the labeling of the momenta in the scattering process: Two original magnons with momenta $\mathbf{k}, \mathbf{q}$ are scattered into the two magnons with momenta $\mathbf{p}$ and $\mathbf{k}+\mathbf{q}-\mathbf{p}$. The momentum of the thermally excited magnons is $\mathbf{q}$. Note that the numerator of the diagram contains the Bose occupation number of the thermal magnons $f\left(\varepsilon_{\mathbf{q}}\right)$, which controls the temperature dependence of the self-energy. In the limit of small temperatures, there is a simplification: Only the thermal magnons from the down-band in the vicinity of the $\Gamma$ point are excited, as is illustrated in Fig. 1(b). The low-temperature expansion allows us to integrate out the thermal magnons $\mathbf{q}$ analytically and reduce Eq. (4) to

$$
\Sigma_{k}^{(2)}(T)=\alpha_{2} T^{2} A \int \frac{d^{2} p}{(2 \pi)^{2}} \frac{\left|v_{\mathbf{k} ; \mathbf{p}}\right|^{2}}{\varepsilon_{\boldsymbol{k}}-\varepsilon_{\mathbf{p}}-\varepsilon_{\mathbf{k}-\mathbf{p}}+i \delta},
$$

where $\alpha_{2}=\left[\pi /\left(6 \sqrt{3} J^{2} S^{2}\right)\right]$ and $v_{\mathbf{k} ; \mathbf{p}}$ is an effective matrix element at low temperature. The remaining sum in Eq. (5) 
is now carried out numerically, and the result is plotted in Fig. 2 for momentum $\boldsymbol{k}$ along a closed path $\Gamma \rightarrow K \rightarrow$ $M \rightarrow \Gamma$ in momentum space. Note that the magnitude of the diagram is set by the overall temperature-dependent prefactor in Eq. (5). We show the self-energy separately for the up- and down-magnon branches in the left and right columns of Fig. 2, correspondingly. The real and imaginary parts of the self-energy have physical significance as the scattering rate and energy renormalization of the magnon excitations, respectively.

First, let us comment on the scattering rate, i.e., the imaginary part of the self-energy, shown in the top row of Fig. 2. The scattering process is on shell; i.e., the energy of the magnons is conserved. Generally, we find that the matrix element $v_{k ; p}$ in the numerator of Eq. (5) is a wellbehaved nonsingular function in momentum space that only slightly modifies the pattern of the self-energy. It is the energy denominator $D_{k ; p}=\varepsilon_{k}-\varepsilon_{p}-\varepsilon_{k-p}$ in Eq. (5) that is the major factor determining the self-energy as a function of momentum. The decay rate displays a complex pattern of peaks and dips. In order to investigate the origin of these features in the self-energy, we examine the behavior of the function $D_{k ; p}$ in momentum space in Ref. [32]. We consistently find that all features in the self-energy correspond to special points of $D_{k: p}$ (e.g., saddle or extremum points). The profile of self-energy in momentum space shown in Fig. 2 strongly depends on the details and parameters of the model and can thus be seen as a unique signature of the honeycomb dispersion $\varepsilon_{k}$. For comparison, we also calculate the scattering rate for the triangular lattice, which can be regarded as a Bravais lattice relative of the honeycomb lattice. We find that the profile of the scattering rate for the triangular lattice is significantly different from Fig. 2. Note that the mirror symmetry
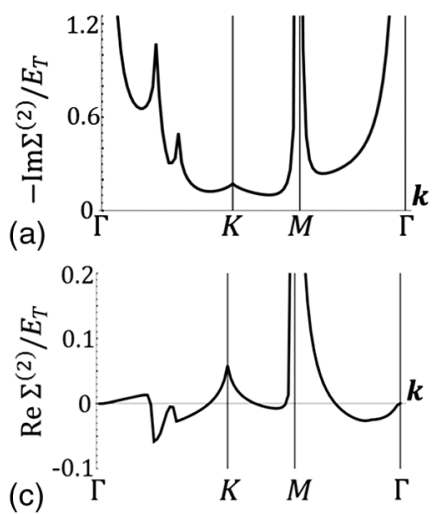

(b)
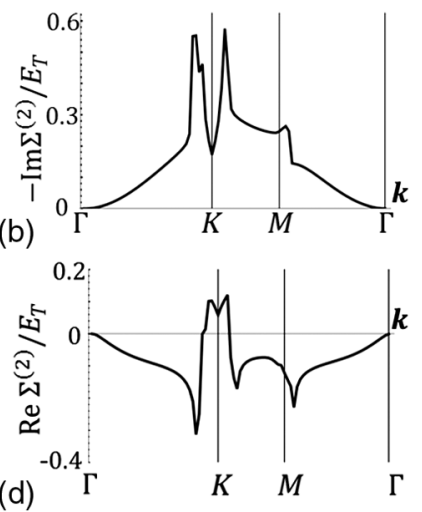

FIG. 2. Normalized decay rate of the (a) up-band and (b) downband. A sequence of van-Hove-like peaks is a unique signature of the Dirac dispersion. Normalized magnon dispersion renormalization for (c) up-band and (d) down-band. The vertical axes are plotted with respect to the temperature-dependent energy unit $E_{T}=T^{2} / J S^{3}$; therefore, all contributions scale with temperature as $T^{2}$.
[33] of the up- and down-magnon branches, i.e., $\varepsilon_{p}^{u}+\varepsilon_{-p}^{d}=6 J S \equiv \varepsilon_{0}^{u}$, leads to the strong peak near the $\Gamma$ point in Fig. 2(a). In terms of Eq. (5), this means that the kinematic constraint appearing in the denominator, $D_{k=0 ; p}=\varepsilon_{0}^{u}-\varepsilon_{p}^{u}-\varepsilon_{-p}^{d}=0$, is satisfied for all $p$, and the integral diverges. Because of the multiband nature of the Hamiltonian, there are multiple scattering channels contributing to each decay process. For example, the scattering rate for the up-band shown in Fig. 2(a) is derived from the two scattering channels $u_{\mathbf{k}} \rightarrow u_{\mathbf{p}}+d_{\mathbf{k}-\mathbf{p}}$ and $u_{\mathbf{k}} \rightarrow d_{\mathbf{p}}+d_{\mathbf{k}-\mathbf{p}}$. The decay rate of the down-band, shown in Fig. 2(b), is determined by the scattering channel $d_{\mathbf{k}} \rightarrow d_{\mathbf{p}}+d_{\mathbf{k}-\mathbf{p}}$, and in the vicinity of the $K$ point, it is sharply suppressed; i.e., the magnon at the Dirac nodal point is a well-defined excitation whose lifetime shrinks rapidly for small excursions away from the $K$ point.

We also plot the real part of the self-energy in the bottom row of Fig. 2 in panel (c) for the up-band and in panel (d) for the down-band. The real and imaginary parts obey the Kramers-Kronig relation, so a peak in the scattering rate corresponds to a feature in the real part of the self-energy. Results in the lower panels of Fig. 2 closely correlate with the upper panels. We pay special attention to the $K$ point, where the Dirac nodal point is located. Here, the values of the real parts of the self-energy for the up and down-bands are equal within the numerical precision. The interaction preserves the degeneracy of the Dirac nodal point. To estimate the effect of renormalization on the magnon energy spectrum, we compare the bare spectrum $\varepsilon_{k}$ and temperature-dependent renormalized spectrum

$$
\begin{aligned}
\varepsilon_{k}^{u, d}(T) & =\varepsilon_{k}^{u, d}+\Sigma_{k}^{(1)}(T)+\Sigma_{k}^{(2)}(T) \\
& =\left(1-\alpha_{1} T^{2}\right) \varepsilon_{k}^{u, d}+\Sigma_{k}^{(2)}(T)
\end{aligned}
$$

in the vicinity of the $K$ point in momentum space in Fig. 3(a). We plot the renormalized spectrum for a given ratio $T / J=3$, which allows us to visualize the effect of renormalization clearly. Interaction leads to renormalization of the energy of the Dirac nodal point, together with a decrease of Dirac velocity in the immediate vicinity of the $K$ point. The latter behavior is in stark contrast to that for the fermionic Dirac materials, where the Coulomb interaction leads to a logarithmic increase of the Dirac velocity $[10,11]$. There are also sharp kinks, marked by the green and blue arrows, in the self-energies, which correspond to equally sharp features in the magnon decay rate and strongly reshape the original linear Dirac dispersion.

We believe that these calculations resolve the old puzzle of the peculiar magnon-band renormalization in $\mathrm{CrBr}_{3}$ observed in the neutron-scattering experiments from the 1970s [8,9]. In Fig. 3(b), we plot the calculated self-energy shift, together with the experimental points from Ref. [8] for $\mathrm{CrBr}_{3}$. The plot shows a relative renormalization of the magnon dispersion of the down-band, which is 

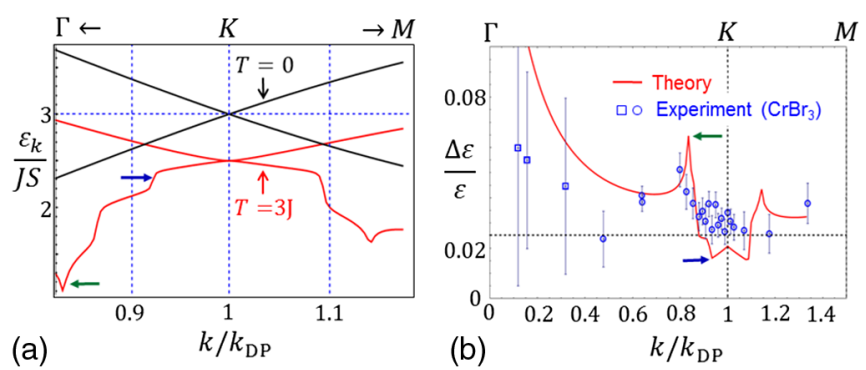

FIG. 3. (a) Temperature-dependent renormalization of the Dirac dispersion near the $K$ point. The bare and renormalized spectra are shown in red and black lines. (b) Temperature-dependent renormalization of the magnon energy spectrum copied from Ref. [34] overlaid with our theory (red). The green and blue arrows in both panels are used as a guide to the eye and point to the corresponding features in the spectrum. In panel (b), the horizontal dashed line represents the energy renormalization due to the constant Hartree term [Eq. (3)]. The momentum $k_{\mathrm{DP}}=4 \pi / 3 \sqrt{3} a$ denotes the position of the Dirac point.

$$
\frac{\Delta \varepsilon_{k}}{\varepsilon_{k}}=\frac{\varepsilon_{k}^{d}(T=6 \mathrm{~K})-\varepsilon_{\mathrm{k}}^{\mathrm{d}}(T=20 \mathrm{~K})}{\varepsilon_{k}^{d}(T=6 \mathrm{~K})}
$$

in the notations of the current work. In the literature, distinct values for the coupling constant are cited: $J=$ $11 \mathrm{~K}$ [35], $J=16 \mathrm{~K}$ [9], and $J=28 \mathrm{~K}$ [36]. We evaluate $\Delta \varepsilon / \varepsilon$ from our theory for the coupling constant $J=18 \mathrm{~K}$, which best fits the experimental data. We then plot $\Delta \varepsilon / \varepsilon$ with the red line and overlay it on the experimental data in Fig. 3(b). Gratifyingly, the theoretical curve and the experimental data agree reasonably well. In particular, the self-energy correction in both experiment and theory undergo rapid evolution in a small region bounded by the peak, marked by the green arrow near $K$.

The strong energy dependence of the self-energy is the result of in-plane interactions and the Dirac spectrum. $\mathrm{CrBr}_{3}$ is a 3D material composed of stacks of weakly coupled, 2D honeycomb layers. To address three-dimensional aspects, one would need to include the interlayer coupling. We expect that the interlayer coupling $J_{z}$ may lead to a weak broadening of peaks in the self-energy shown in Fig. 2, but the magnitude of the effect is small since the interlayer coupling $J_{z}$ is much smaller than the intralayer coupling $J_{z} \ll J[9]$.

The results obtained in this work are universal and apply to other types of bosonic Dirac materials. The profile of $\operatorname{Re} \Sigma(\mathbf{k})$ in momentum space in Fig. 2 is determined predominantly by energy denominators and less so by the matrix elements in Eqs. (4) and (5). Thus, the momentum dependence of these quantities could be viewed as a unique signature of the honeycomb dispersion. Therefore, our findings should be highly relevant to other bosonic honeycomb systems, e.g., photonic crystals [2,3,37-41].

\section{RESULTS: SURFACE STATES IN A 3D MODEL}

Dirac nodes have profound consequences for the topological surface states that are analogous to Fermi nodal lines. Because the honeycomb lattice is bipartite, it is a prime candidate to possess topological Shockley edge states (otherwise known as Su-Schrieffer-Heeger states). The three-dimensional crystal structure of $\mathrm{CrBr}_{3}$ shown in Fig. 4(a) consists of the honeycomb $\mathrm{Cr}$ layers stacked in the order that is an analog of the ABC-stacked graphene layers, which have received a lot of attention because of the unusual spectrum and edge states [24-26]. We assume the simplest model in which the honeycomb layers are coupled via the vertical bonds with a strength $J_{z}$, as illustrated in Fig. 4(a). Thus, the corresponding freemagnon Hamiltonian becomes

$$
H_{0}(\mathbf{k})=J S\left(\begin{array}{cc}
3+\gamma_{z} & -\gamma_{k}-\gamma_{z} e^{i k_{z}} \\
-\gamma_{k}^{*}-\gamma_{z} e^{-i k_{z}} & 3+\gamma_{z}
\end{array}\right) .
$$

In comparison with Hamiltonian (1), Eq. (8) acquires extra terms proportional to $\gamma_{z}=J_{z} / J$. Let us examine the Dirac points given by the equation $\gamma_{k}+\gamma_{z} e^{i k_{z}}=0$ as well as the dispersion in the $k_{z}$ direction. For small $\gamma_{z}$, the solution gives helical contours winding around the corners of the Brillouin zone as shown in Fig. 4(b). This nodal line, i.e., a line where the magnon branches are degenerate, retains the topological properties of the usual Dirac point. In particular, the nodal line has a topological Berry phase,

$$
\oint d \mathbf{k}\left\langle\Psi_{\mathbf{k}} \mid i \partial_{\mathbf{k}} \Psi_{\mathbf{k}}\right\rangle=\pi
$$

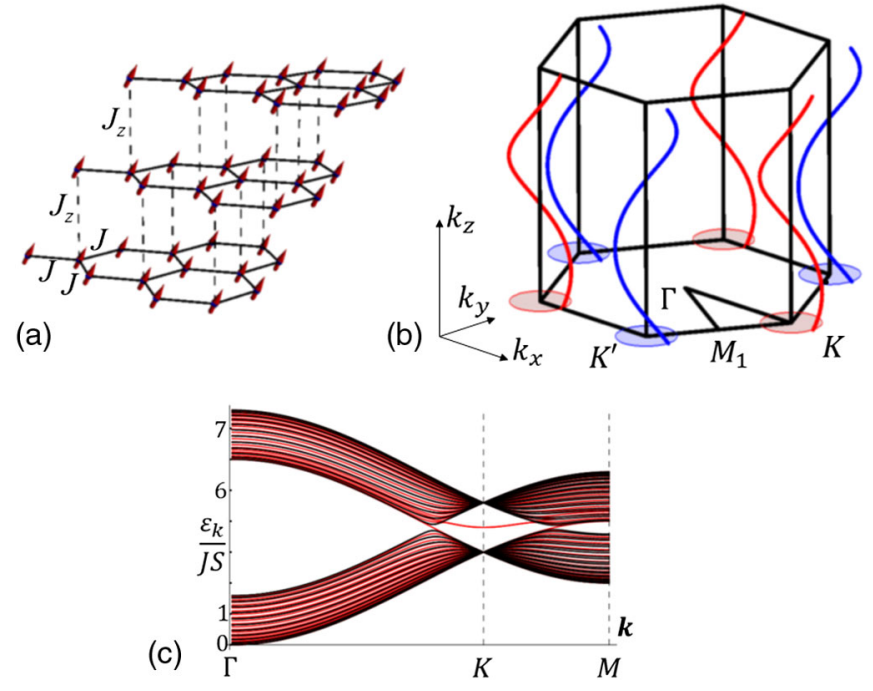

FIG. 4. (a) The 3D lattice structure of $\mathrm{CrBr}_{3}$. The honeycomb layers are composed of $\mathrm{Cr}$ atoms and are stacked in $\mathrm{ABC}$ order. (b) The band structure: Dirac nodal lines wind around the corners of the Brillouin zone. (c) Magnon energy spectrum for a 25-layer slab of a three-dimensional material. Results for open vs closed boundary conditions are shown with red and black lines. 
which leads to flat band surface states (in the fermionic case) given by the projection of the nodal line onto the $2 \mathrm{D}$ momentum space [24-26] as illustrated in Fig. 4(b).

Now, we evaluate the surface states in the 3D model discussed. Although the surface states are calculated at the single-particle level, it turns out that the surface magnons differ from their fermionic analogs. We compute the magnon dispersion in a slab geometry of $N=25$ layers and plot the result in Fig. 4(c). The figure contains the spectrum for the closed (in black) and open (in red) boundary conditions. In this representation, the red lines unmatched by the black lines correspond to the surface states.

One can observe the magnon surface states appearing in the vicinity of the $K$ point, which agree with Fig. 4(b). However, in contrast to what occurs for the analogous fermionic model, the magnon surface states are clearly dispersive. To explain this, we recall the original Heisenberg Hamiltonian $\mathcal{H}=-J \sum_{\langle i j\rangle} \boldsymbol{S}_{i} \cdot \boldsymbol{S}_{j}$ and the Holstein-Primakoff transformation, e.g., for the $z$ th component of the spin operators $S_{i}^{z}=S-a_{i}^{\dagger} a_{i}$. Observe that intersite coupling in the Heisenberg Hamiltonian generates an on-site potential in the bosonic language, i.e., $-J S_{i}^{z} S_{j}^{z}=-J S^{2}+J S a_{i}^{\dagger} a_{i}+J S b_{i}^{\dagger} b_{i}+\mathcal{O}\left(a^{4}\right)(i \neq j)$. For the bulk, this procedure generates the diagonal terms in the Hamiltonians (1) and (8). For the surface, inspection of Fig. 4(a) shows that one sublattice-we assume it is $A-$ will be coupled (to the $B$ sublattice) in the layer immediately below, but not the other. This generates an extra diagonal surface term $H_{\text {surface }}=-J_{z} S a^{\dagger} a$ for the $A$ but not the $B$ sublattice, in addition to the off-diagonal terms that allow hopping from the $A$ sublattice of the surface to the $B$ sublattice. In the absence of the diagonal surface term, we obtain dispersionless topological surface states that are identical to the fermionic analogs (see, e.g., Ref. [26]). Note that the decay length $\xi_{\mathbf{k}} \propto 1 / \Delta_{\mathbf{k}}$ of this surface state $\Psi_{\mathbf{k}}(z) \sim \exp \left(-z / \xi_{\mathbf{k}}\right)$ in the $z$ direction is inversely proportional to the bulk gap $\Delta_{\mathbf{k}}$, where $\mathbf{k}=\left(k_{x}, k_{y}\right)$ is a good quantum number for a surface cleaved parallel to the layers. Qualitatively, the dispersion of the magnon surface states can then be understood by treating the surface term as a perturbation. Already, the first-order perturbation theory produces a k-dependent dispersion of the surface states, $\varepsilon_{\text {surface }}(\mathbf{k})=\left\langle\Psi_{\mathbf{k}}(z)\left|H_{\text {surface }}\right| \Psi_{\mathbf{k}}(z)\right\rangle$. Indeed, the nonperturbative exact diagonalization of the bosonic Hamiltonian, together with the surface term, confirms that the surface state acquires a strong dispersion (as shown in Ref. [32]). We further relax the interplanar Heisenberg coupling $J_{z}$ as the surface is approached; Fig. 4(c) is plotted for a reduced coupling $J_{z}^{\text {surface }}=0.5 J_{z}^{\text {bulk }}$ between the surface and penultimate layers. We also note that an earlier study investigated the effect of edge perturbations in topological magnonic media [20]. Our discussion extends the previous analysis of the edge states in 2D photonic materials. We also mention that the mechanism described for generating the dispersion of the surface states is universal and should be relevant to other types of bosonic Dirac materials, e.g., topological phononic and photonic systems [2,3,37-42].

We also mention several other works [21,22], which were done in parallel with our work, exploring the nodal lines in magnonic media. Reference [22] discussed the existence and consequences of the nodal line in a quasitwo-dimensional setting. Reference [21] predicted the existence of the magnon nodal lines in a complex fourband model in anisotropic pyrochlore ferromagnets and revealed a strong dependence of the surface states on the crystal termination. In contrast, our paper discusses the nodal lines in the simplest two-band model in a threedimensional setting. Our work is important in the context of realizing a bosonic ABC-stacked "graphite." Note that the analogous $\mathrm{ABC}$-stacked "fermionic" graphite is highly sought after [43] but is not realized naturally (AB "Bernal" stacking is stable). We propose that the nodal lines discussed in this section could be readily realized in $\mathrm{CrBr}_{3}$, which has natural $\mathrm{ABC}$ stacking. The corresponding magnonic surface states may serve as a pairing "glue" for exotic orders on the surface of these materials.

\section{CONCLUSION}

Noninteracting particles in a $2 \mathrm{D}$ honeycomb lattice exhibit Dirac excitations regardless of the statistics. We consider the difference between bosonic and fermionic excitations on such lattices. Two key features of the bosonic Dirac material were identified as important: (i) Interacting bosons lead to a nondivergent velocity renormalization near the Dirac points. In contrast, Coulomb interactions between fermions lead to a logarithmic correction of the Dirac velocity. (ii) The non-Bravais nature of the honeycomb lattice structure leads to significant modifications of the spin-wave interactions. More generally, non-Bravais lattices, which entail multicomponent wave functions for quasiparticles, provide a route to Dirac quasiparticles and distinct surface states. (iii) We found topological surface states in $\mathrm{CrBr}_{3}$ that are the bosonic analog of Shockley edge and surface states (also known as the Su-Schrieffer-Heeger states).

Our results are important because of the ferromagnetic $\mathrm{Cr}$ trihalides. Magnetic excitations in honeycomb ferromagnetic layers, the building blocks of the $\mathrm{Cr}$ trihalides, possess Dirac nodes. Half a century ago, neutron-scattering experiments observed an anomalous momentum-dependent renormalization of the magnon spectrum in $\mathrm{CrBr}_{3}$ near the Dirac points [8,9]. By evaluating the self-energy due to the magnon-magnon interactions, we obtained a good agreement with data from Ref. [8] and have thus resolved a nearly 50-year-old puzzle.

The honeycomb ferromagnets are the conceptually simplest systems where the magnonic Dirac cones could be found. Therefore, much of the novel Dirac physics already developed in graphene can be compared and tested 
in the magnonic Dirac systems, which are readily available in chromium trihalides. Although the single-particle Hamiltonians in the bosonic and fermionic systems appear to be superficially analogous, the effect of the quasiparticle statistics is decisive. As shown in our work, interaction effects and topological surface states are substantially different in the magnonic Dirac materials, so there are many opportunities for new experiments. We envision that the magnonic Dirac materials could become important as platforms for magnon transport and as a basic building block to construct topological magnetic media. Pumped magnonic Dirac matter and Bose-Einstein condensation [44] is also very intriguing to pursue.

Given the large diversity of transition metal trihalides $[36,45]$, metamaterials generated via top-down and bottomup nanotechnology, and cold atom systems that can implement bosonic Hamiltonians for honeycomb and other nonBravais lattices, our predictions are highly relevant and can be extensively checked using modern spectroscopic methods. For example, neutron scattering has advanced greatly over the last five decades, so we anticipate new work to measure the lifetimes as well as self-energies of spin waves for honeycomb magnets. There are also potential applications of the surface states, which we have discovered, to the emerging area of "magnon spintronics" or "magnonics" [46], for which distinct surface spin waves would be advantageous because they avoid bulk dissipation.

Recent experimental observation of single-layer honeycomb ferromagnetism $[27,28]$ in $\mathrm{CrI}_{3}$ and other materials highlights the important role the Dirac nodes will play in bosonic Dirac materials realized in van der Waals magnetic structures.

\section{ACKNOWLEDGMENTS}

This work is supported by U.S. Department of Energy Grant No. BES E3B7, Villum Center for Dirac Materials, Grant No. ERC DM-321031, Grant No. KAW-2013-0096, and Dr. Max Rössler, the Walter Haefner Foundation, and the ETH Zurich Foundation. H. A. acknowledges the Knut and Wallenberg Foundation for financial support (Grant No. KAW-2013-0020) and the Villum Foundation. A. V. B acknowledges the hospitality and support from the Institute for Theoretical Studies, ETH Zurich, and from Director's Office, Paul Scherrer Institute, where part of this work was carried out. We are grateful to A. Chernyshev and P. Hofmann for useful discussions.

[1] A. H. Castro Neto, F. Guinea, N. M. R. Peres, K. S. Novoselov, and A. K. Geim, The Electronic Properties of Graphene, Rev. Mod. Phys. 81, 109 (2009).

[2] F. D. M. Haldane and S. Raghu, Possible Realization of Directional Optical Waveguides in Photonic Crystals with Broken Time-Reversal Symmetry, Phys. Rev. Lett. 100, 013904 (2008).
[3] T. Ma, A. B. Khanikaev, S. H. Mousavi, and G. Shvets, Guiding Electromagnetic Waves around Sharp Corners: Topologically Protected Photonic Transport in Metawaveguides, Phys. Rev. Lett. 114, 127401 (2015).

[4] G. Weick, C. Woollacott, W. L. Barnes, O. Hess, and E. Mariani, Dirac-like Plasmons in Honeycomb Lattices of Metallic Nanoparticles, Phys. Rev. Lett. 110, 106801 (2013).

[5] S. Banerjee, J. Fransson, A. M. Black-Schaffer, H. Ågren, and A. V. Balatsky, Granular Superconductor in a Honeycomb Lattice as a Realization of Bosonic Dirac Material, Phys. Rev. B 93, 134502 (2016).

[6] J. Fransson, A. M. Black-Schaffer, and A. V. Balatsky, Magnon Dirac Materials, Phys. Rev. B 94, 075401 (2016).

[7] S. A. Owerre, A First Theoretical Realization of Honeycomb Topological Magnon Insulator, J. Phys. Condens. Matter 28, 386001 (2016).

[8] W. B. Yelon and R. Silberglitt, Renormalization of LargeWave-Vector Magnons in Ferromagnetic $\mathrm{CrBr}_{3}$ Studied by Inelastic Neutron Scattering: Spin-Wave Correlation Effects, Phys. Rev. B 4, 2280 (1971).

[9] E. J. Samuelsen, R. Silberglitt, G. Shirane, and J. P. Remeika, Spin Waves in Ferromagnetic $\mathrm{CrBr}_{3}$ Studied by Inelastic Neutron Scattering, Phys. Rev. B 3, 157 (1971).

[10] J. Gonzalez, F. Guinea, and M. A. H. Vozmediano, NonFermi Liquid Behavior of Electrons in the Half-Filled Honeycomb Lattice (A Renormalization Group Approach), Nucl. Phys. B424, 595 (1994).

[11] D. C. Elias, R. V. Gorbachev, A. S. Mayorov, S. V. Morozov, A. A. Zhukov, P. Blake, L. A. Ponomarenko, I. V. Grigorieva, K. S. Novoselov, F. Guinea, and A. K. Geim, Dirac Cones Reshaped by Interaction Effects in Suspended Graphene, Nat. Phys. 7, 701 (2011).

[12] F. Bloch, Zur Theorie des Ferromagnetismus, Z. Phys. 61, 206 (1930).

[13] S. V. Akhiezer, A. I. Bar'yakhtar, and V. G. Peletminskii, Spin Waves (Nauka, Moscow, in Russian, 1967).

[14] V. L. Safonov, Nonequilibrium Magnons: Theory, Experiment and Applications, 1st ed. (Wiley-VCH, New York, 2012).

[15] A. S. Borovik-Romanov and S. K. Sinha, Spin Waves and Magnetic Excitations (North-Holland, Amsterdam, 1988).

[16] F. J. Dyson, General Theory of Spin-Wave Interactions, Phys. Rev. 102, 1217 (1956).

[17] F. J. Dyson, Thermodynamic Behavior of an Ideal Ferromagnet, Phys. Rev. 102, 1230 (1956).

[18] M. Bloch, Magnon Renormalization in Ferromagnets Near the Curie Point, Phys. Rev. Lett. 9, 286 (1962).

[19] L. Zhang, J. Ren, J.-S. Wang, and B. Li, Topological Magnon Insulator in Insulating Ferromagnet, Phys. Rev. B 87, 144101 (2013).

[20] A. Mook, J. Henk, and I. Mertig, Edge States in Topological Magnon Insulators, Phys. Rev. B 90, 024412 (2014).

[21] A. Mook, J. Henk, and I. Mertig, Magnon Nodal-Line Semimetals and Drumhead Surface States in Anisotropic Pyrochlore Ferromagnets, Phys. Rev. B 95, 014418 (2017).

[22] S. A. Owerre, Dirac Magnon Nodal Loops in Quasi-2D Quantum Magnets, Sci. Rep. 7, 6931 (2017).

[23] K. Nakata, J. Klinovaja, and D. Loss, Magnonic Quantum Hall Effect and Wiedemann-Franz Law, Phys. Rev. B 95, 125429 (2017). 
[24] T. T. Heikkilä and G. E. Volovik, Dimensional Crossover in Topological Matter: Evolution of the Multiple Dirac Point in the Layered System to the Flat Band on the Surface, JETP Lett. 93, 59 (2011).

[25] P. Delplace, D. Ullmo, and G. Montambaux, Zak Phase and the Existence of Edge States in Graphene, Phys. Rev. B 84, 195452 (2011).

[26] S. S. Pershoguba and V. M. Yakovenko, Shockley Model Description of Surface States in Topological Insulators, Phys. Rev. B 86, 075304 (2012).

[27] C. Gong, L. Li, Z. Li, H. Ji, A. Stern, Y. Xia, T. Cao, W. Bao, C. Wang, Y. Wang, Z. Q. Qiu, R. J. Cava, S. G. Louie, J. Xia, and X. Zhang, Discovery of Intrinsic Ferromagnetism in Two-Dimensional van der Waals Crystals, Nature (London) 546, 265 (2017).

[28] B. Huang, G. Clark, E. Navarro-Moratalla, D. R. Klein, R. Cheng, K. L. Seyler, D. Zhong, E. Schmidgall, M. A. McGuire, D. H. Cobden, W. Yao, D. Xiao, P. JarilloHerrero, and X. Xu, Layer-Dependent Ferromagnetism in a van der Waals Crystal Down to the Monolayer Limit, Nature (London) 546, 270 (2017).

[29] M. E. Zhitomirsky and A. L. Chernyshev, Colloquium: Spontaneous Magnon Decays, Rev. Mod. Phys. 85, 219 (2013).

[30] P. A. Maksimov and A. L. Chernyshev, Field-Induced Dynamical Properties of the XXZ Model on a Honeycomb Lattice, Phys. Rev. B 93, 014418 (2016).

[31] J. L. Lado and J. Fernández-Rossier, On the Origin of Magnetic Anisotropy in Two Dimensional $\mathrm{CrI}_{3}$, 2D Mater. 4, 035002 (2017).

[32] See Supplemental Material at http://link.aps.org/ supplemental/10.1103/PhysRevX.8.011010 for detailed discussion of microscopic calculations, different scattering terms, comparison with the triangular ferromagnet, topology and emergence of the surface states.

[33] A. L. Chernyshev and P. A. Maksimov, Damped Topological Magnons in the Kagome-Lattice Ferromagnets, Phys. Rev. Lett. 117, 187203 (2016).

[34] T. O. Wehling, A. M. Black-Schaffer, and A. V. Balatsky, Dirac Materials, Adv. Phys. 63, 1 (2014).

[35] H. L. Davis and A. Narath, Spin-Wave Renormalization Applied to Ferromagnetic $\mathrm{CrBr}_{3}$, Phys. Rev. 134, A433 (1964).
[36] H. Wang, F. Fan, S. Zhu, and H. Wu, Doping Enhanced Ferromagnetism and Induced Half-Metallicity in $\mathrm{CrI}_{3}$ Monolayer, Europhys. Lett. 114, 47001 (2016).

[37] G.-W. Chern and A. Saxena, PT-Symmetric Phase in Kagome-Based Photonic Lattices, Opt. Lett. 40, 5806 (2015).

[38] Z. Wang, Y. D. Chong, J. D. Joannopoulos, and M. Soljačić, Reflection-Free One-Way Edge Modes in a Gyromagnetic Photonic Crystal, Phys. Rev. Lett. 100, 013905 (2008).

[39] S. Raghu and F. D. M. Haldane, Analogs of Quantum-HallEffect Edge States in Photonic Crystals, Phys. Rev. A 78, 033834 (2008).

[40] Z. Wang, Y. Chong, J. D. Joannopoulos, and M. Soljacic, Observation of Unidirectional Backscattering-Immune Topological Electromagnetic States, Nature (London) 461, 772 (2009).

[41] J. D. Joannopoulos, P. R. Villeneuve, and S. Fan, Photonic Crystals: Putting a New Twist on Light, Nature (London) 386, 143 (1997).

[42] O. Stenull, C. L. Kane, and T. C. Lubensky, Topological Phonons and Weyl Lines in Three Dimensions, Phys. Rev. Lett. 117, 068001 (2016).

[43] T. T. Heikkilä and G. E. Volovik, Flat Bands as a Route to High-Temperature Superconductivity in Graphite, in Basic Physics of Functionalized Graphite, Springer Series in Materials Science, edited by P.D. Esquinazi (Springer, New York, 2016), Vol. 244, pp. 123-143.

[44] S. O. Demokritov, V. E. Demidov, O. Dzyapko, G. A. Melkov, A. A. Serga, B. Hillebrands, and A. N. Slavin, Bose-Einstein Condensation of Quasi-equilibrium Magnons at Room Temperature under Pumping, Nature (London) 443, 430 (2006).

[45] W.-B. Zhang, Q. Qu, P. Zhu, and C.-H. Lam, Robust Intrinsic Ferromagnetism and Half Semiconductivity in Stable Two-Dimensional Single-Layer Chromium Trihalides, J. Mater. Chem. C 3, 12457 (2015).

[46] A. V. Chumak, V. I. Vasyuchka, A. A. Serga, and B. Hillebrands, Magnon Spintronics, Nat. Phys. 11, 453 (2015). 